\title{
Analysis of Rainfall and Other Weather Parameters under Climatic Variability of Parbhani (2011-2015)
}

\author{
Pooran Pragnya, Tapre Praveen and B. W. Bhuibhar*
}

College of Agricultural Engineering and Technology, Parbhani (MS), India

*Corresponding author

\begin{tabular}{|c|}
\hline Keywords \\
\hline $\begin{array}{l}\text { Rainfall, Temperature, } \\
\text { Meteorological } \\
\text { parameters, climatic } \\
\text { variability, monthly basis }\end{array}$ \\
\hline Article Info \\
\hline $\begin{array}{l}\text { Accepted: } \\
20 \text { May } 2018 \\
\text { Available Online: } \\
\text { 10 June } 2018\end{array}$ \\
\hline
\end{tabular}

\section{Keywords}

Rainfall, Temperature,

Meteorological

variability, monthly basis

Article Info

Accepted:

Available Online:

10 June 2018

\section{A B S T R A C T}

Parbhani is intersected by $19^{\circ} 08^{\prime} \mathrm{N}$ latitude and $76^{\circ} 50^{\prime} \mathrm{E}$ longitude. The average annual rainfall at Parbhani station is $750-800 \mathrm{~mm}$, which is mostly received from south-west monsoon. The weather parameters include mean monthly rainfall, average weekly rainfall (AWR) and average annual rainfall. All other meteorological parameters were analysed on monthly basis to study the climatic variability. The maximum average weekly rainfall of Parbhani was $154 \mathrm{~mm}$ recorded in 2013 during $29^{\text {th }}$ meteorological week. The July month of 2013 recorded maximum rainfall of $421.2 \mathrm{~mm}$. Monsoon season of 2013 recorded maximum average rainfall with $1017.1 \mathrm{~mm}$ when compared with all other seasons. Annual average maximum rainfall of $1207.3 \mathrm{~mm}$ was observed in 2013 and minimum average annual rainfall of $569.7 \mathrm{~mm}$ was recorded during 2014 with a mean average annual rainfall of $743.5 \mathrm{~mm}$. May month of 2013 recorded maximum average monthly temperature of $42.75^{\circ} \mathrm{C}$ and minimum average monthly temperature of $9.51^{\circ} \mathrm{C}$ was recorded in January, 2011 when compared with all other months of entire study period from 2011 to 2015.Maximum average monthly relative humidity of $91.17 \%$ was recorded in September month of 2012 while monthly minimum RH was recorded in March month with $12.39 \%$ of the same year. May month of 2013 recorded maximum evaporation rate with $15.15 \mathrm{~mm}$ while August month of the same year recorded minimum evaporation rate of $3.71 \mathrm{~mm}$ in the entire study period. Maximum average monthly bright sun shine hours were recorded in April, 2011 with $10.58 \mathrm{hrs}$ and minimum was recorded in the month of July, 2013 with $2.71 \mathrm{hrs}$. June month of 2011 recorded maximum average monthly wind speed of $9.05 \mathrm{kmph}$ while lowest was observed in December month of the same year with $2.56 \mathrm{kmph}$.

\section{Introduction}

Rainfed agriculture is a highly risky business. Uncertain precipitation is a major constraint to crop production especially when it occurs at an unexpected time and problems become aggravated if dry conditions prevail for a longer time (Jestinos Mzezewa1 et al., 2010). The major source of water available either for agriculture or for human consumption is obtained from the rain. Rainfall is one of the most important natural inputs in hydrologic process. South -West monsoon, North- East monsoon, cyclonic depressions and local storms contribute to rainfall in different degrees in various rainfall regions of the country. Due to high temperature of summer, the moisture - laden South-West monsoon 
originates from the vast expanse of the Indian Ocean and enters the Indian sub-continent from the south - West. These south - West monsoonic winds increase from June to July and begin to weaken in September especially in north India.

The occurrence of certain amounts of rainfall at crucial times can determine the successes of failure of crop it depends on the the duration of stress period (Biswas, 1981). The average annual rainfall over the plains of India is $119.4 \mathrm{~cm}$ and the average for the lands of the world put together is only $70 \mathrm{~cm}$ per year. Though India has 1.7 times the average annual rainfall of whole world, its agricultural production is at low level. The average annual rainfall of Maharastra is $120 \mathrm{~cm}$ whereas rainfall of Marathwada varies between $550 \mathrm{~mm}$ to $1100 \mathrm{~mm}$ with an average of $774 \mathrm{~mm}$. The precipitation of Parbhani varies from 700$800 \mathrm{~mm}$ per year with an average of $745 \mathrm{~mm}$. Precipitation is very uncertain in this region and sometimes suffers from severe droughts.

Under such circumstances for increasing crop productivity, better crop planning for carrying out the different agricultural activities at proper time could be beneficial (Cannarozzo et al., 2006). For better crop planning, information related to the dates of onset of effective monsoon, evaporation data depending on sunshine hours, temperature during the different developmental stages of the crops plays an important role.

Keeping these points in view, a special problem was undertaken with the following objectives:

To analyze monthly data of various meteorological parameters viz, Rainfall, wind speed, maximum and minimum temperature, evaporation rate, bright sunshine hours and morning and evening relative humidity of last five years for Parbahni.
To analyze rainfall data on weekly, monthly, seasonally and yearly basis of last five years for Parbhani

\section{Materials and Methods}

\section{Features of study area}

The area is located in semi-arid region of Maharastra state. Parbhani is intersected by $19^{0} 08^{\prime} \mathrm{N}$ latitude and $76^{\circ} 50$ ' E longitude. It comes under moderate rainfall zone at an altitude of $409 \mathrm{~m}$ above mean sea level. The average annual rainfall at Parbhani station is $750-800 \mathrm{~mm}$, which is mostly received from south-west monsoon. Monsoon is generally concentrated over the months of June to September. The average annual air temperature is about $25.95^{\circ} \mathrm{C}$ and the coldest and warmest monthly temperatures are $9.5^{\circ} \mathrm{C}$ and $42^{\circ} \mathrm{C}$ in December and May respectively.

\section{Data collection and analysis}

The methodology consists of collection of long term daily weather data such in respect of rainfall $(\mathrm{mm})$ and other meteorological parameters. The daily rainfall data during 2011-2015 was collected from meteorology department, Parbhani and analyzed on annual basis. The daily rainfall data collected from meteorological department was concised to annual basis from 2011 - 2015 along with deviation. The number of rainy days was also calculated yearly and was presented graphically. For monthly temporal analysis the daily data was averaged or sum up into month from January to December and grouped into years for the period of 2011 -2015.whereas for seasonal rainfall analysis monthly data was grouped into three seasons as summer (MarchMay), monsoon (June - September) and winter (October - February). The parameters were then analyzed using statistical tools such as Mean, Standard deviation and Coefficient of variance. 


\section{Results and Discussion}

Record of daily rainfall data for 5 years for Parbhani station was obtained from the Department of Agricultural Meteorology, V.N.M.K.V, Parbhani. Data were analyzed to determine rainfall characteristics influencing agricultural operations and irrigation management.

The characteristics include mean monthly rainfall, average weekly rainfall (AWR) and average annual rainfall. All other meteorological parameters such as maximum temperature, minimum temperature, wind speed, morning relative humidity, evening relative humidity, evaporation, and bright sunshine hours of each day from 2011-2015 were collected. These parameters were analysed on monthly basis to study the climatic variability.

\section{Weekly Analysis of Rainfall Data}

The daily rainfall data of the station was grouped into weeks from January $1^{\text {st }}$ to December $31^{\text {st }}$ according to meteorological weeks. The weekly rainfall data for the entire period from 2011 to 2015 are tabulated in table 1 .

Table 1 Indicates that $29^{\text {th }}$ meteorological week of 2013 received highest rainfall (154 $\mathrm{mm})$ and had highest standard of deviation (76.10) when compared with all other meteorological weeks of entire study period from 2011 to 2015. Highest coefficient of variance was observed in $17^{\text {th }}$ week. Figure 1 depicts the graphical presentation of average weekly rainfall data

\section{Monthly Average Rainfall}

The daily rainfall data of the Parbhani station was grouped into months from January to December. The monthly average rainfall data for the entire period from 2011 to 2015 are tabulated in table 2.

The table 2 Revealed that the July month of 2013 had highest rainfall of $421.2 \mathrm{~mm}$ with highest standard deviation of 156.28 when compared with all other months of the entire period from $2011-2015$.

Highest coefficient of Variance of $223.68 \%$ was observed during December month. A similar finding was reported by Mohd Asim and Satyendra Nath (2015).

\section{Monthly Maximum Average Temperature}

Daily maximum temperature data was collected and analyzed into monthly basis. The table 3 depicts the monthly average maximum temperature of Parbhani from 20112015

The table 3 revealed that May month of 2013 recorded maximum average monthly temperature of $42.75^{\circ} \mathrm{C}$ when compared with all other months of entire study period from 2011 to 2015. Highest coefficient of Variance was observed in July month, which was about $6.72 \%$.

\section{Monthly Minimum Average Temperature}

Daily minimum temperature data was collected and analyzed into monthly basis. The table 4.3 depicts the monthly average minimum temperature of Parbhani from 20112015.

From the table 4 it was observed that January month of 2011 had minimum average monthly temperature when compared with all other months of last five years.

It recorded $9.51^{\circ} \mathrm{C}$. November month recorded highest coefficient of variance which was about $15.82 \%$. 
Table.1 Weekly rainfall analysis of last five years data for Parbhani (in mm)

\begin{tabular}{|c|c|c|c|c|c|c|c|c|c|}
\hline \multirow[t]{2}{*}{ WK } & \multirow[t]{2}{*}{ Period } & \multicolumn{5}{|c|}{ Year } & \multirow[t]{2}{*}{ Mean } & \multirow[t]{2}{*}{ S.D } & \multirow[t]{2}{*}{$\mathrm{CV}(\%)$} \\
\hline & & 2011 & 2012 & 2013 & 2014 & 2015 & & & \\
\hline 01 & 01-07 Jan. & 0.0 & 0.0 & 1.0 & 0.0 & 9.2 & 2.04 & 4.06 & 199.02 \\
\hline 02 & 08-14 Jan. & 0.0 & 0.0 & 0.0 & 0.0 & 0.0 & 0.0 & 0.0 & 0.0 \\
\hline 03 & 15-21 Jan. & 0.0 & 0.0 & 0.0 & 0.0 & 0.0 & 0.0 & 0.0 & 0.0 \\
\hline 04 & 22-28 Jan. & 0.0 & 0.0 & 0.0 & 0.0 & 0.0 & 0.0 & 0.0 & 0.0 \\
\hline 05 & 29-04 Feb. & 0.0 & 0.0 & 0.0 & 0.0 & 0.0 & 0.0 & 0.0 & 0.0 \\
\hline 06 & 05-11 Feb. & 0.0 & 0.0 & 0.0 & 0.0 & 0.0 & 0.0 & 0.0 & 0.0 \\
\hline 07 & 12-18 Feb. & 0.0 & 0.0 & 18.5 & 0.0 & 0.0 & 03.7 & 8.27 & 223.51 \\
\hline 08 & 19-25 Feb. & 0.0 & 0.0 & 4.2 & 8.0 & 0.0 & 2.44 & 3.06 & 125.41 \\
\hline 09 & 26-04 Mar. & 0.0 & 0.0 & 0.0 & 22.8 & 24.3 & 9.42 & 12.91 & 137.05 \\
\hline 10 & 05-11 Mar. & 0.0 & 0.0 & 0.0 & 70.7 & 16.6 & 17.46 & 30.62 & 175.37 \\
\hline 11 & 12-18 Mar. & 0.0 & 0.0 & 8.6 & 0.0 & 0.0 & 1.72 & 3.85 & 223.84 \\
\hline 12 & 19-25 Mar. & 1.0 & 0.0 & 0.0 & 0.0 & 0.0 & 0.2 & 0.45 & 225.00 \\
\hline 13 & 26-01 Apr. & 0.7 & 0.0 & 0.0 & 0.0 & 0.0 & 0.14 & 0.31 & 221.43 \\
\hline 14 & 02-28 Apr & 0.0 & 0.0 & 0.0 & 0.0 & 0.0 & 0.0 & 0.0 & 0.0 \\
\hline 15 & 09-15 Apr & 18.6 & 0.0 & 0.0 & 0.5 & 24.6 & 8.74 & 11.93 & 136.50 \\
\hline 16 & 16-22 Apr & 4.2 & 0.6 & 0.0 & 3.7 & 67.2 & 15.14 & 29.16 & 192.60 \\
\hline 17 & 23-29 Apr. & 0.6 & 0.0 & 0.0 & 0.0 & 0.0 & 0.12 & 0.27 & 225.00 \\
\hline 18 & 30-06 May & 15.2 & 0.0 & 0.0 & 8.6 & 22.8 & 9.32 & 9.88 & 106.09 \\
\hline 19 & 07-13 Мау & 0.5 & 0.8 & 0.0 & 1.0 & 2.0 & 0.86 & 0.74 & 86.05 \\
\hline 20 & 14-20 Мay & 0.0 & 0.6 & 0.0 & 4.2 & 0.0 & 0.96 & 1.83 & 190.63 \\
\hline 21 & 21-27 May & 0.7 & 4.0 & 0.0 & 10.0 & 0.0 & 2.94 & 4.28 & 145.58 \\
\hline 22 & 28-03 June & 3.0 & 0.0 & 0.0 & 12.5 & 0.0 & 3.10 & 5.41 & 174.51 \\
\hline 23 & 04-10 June & 11.8 & 8.3 & 107.5 & 6.0 & 27.0 & 32.12 & 42.93 & 133.66 \\
\hline 24 & 11-17 June & 0.0 & 1.6 & 29.2 & 13.0 & 61.1 & 20.98 & 25.28 & 120.50 \\
\hline 25 & 18-24 June & 5.6 & 58.2 & 24.0 & 24.5 & 37.5 & 29.96 & 19.45 & 64.92 \\
\hline 26 & 25-01 July & 15.9 & 14.4 & 41.8 & 0.0 & 0.0 & 14.42 & 17.09 & 118.52 \\
\hline 27 & 02-08 July & 64.2 & 37.3 & 80.1 & 0.5 & 5.0 & 37.42 & 35.19 & 94.04 \\
\hline 28 & 09-15 July & 75.4 & 27.2 & 107.4 & 73.8 & 0.0 & 56.76 & 42.71 & 75.25 \\
\hline 29 & 16-22 July & 17.8 & 134.9 & 154.0 & 1.5 & 0.6 & 61.76 & 76.10 & 123.22 \\
\hline 30 & 23-29 July & 93.7 & 21.0 & 51.5 & 26.2 & 8.0 & 40.08 & 33.88 & 84.53 \\
\hline 31 & 30-05 Aug & 65.0 & 18.9 & 77.5 & 32.4 & 19.8 & 42.72 & 26.95 & 63.09 \\
\hline 32 & 06-12 Aug & 2.8 & 5.9 & 19.7 & 16.7 & 28.8 & 14.78 & 10.57 & 71.52 \\
\hline 33 & 13-19 Aug & 39.3 & 4.8 & 50.2 & 0.0 & 21.0 & 23.06 & 21.62 & 93.76 \\
\hline 34 & 20-26 Aug & 61.3 & 35.4 & 9.3 & 59.8 & 13.6 & 35.88 & 24.60 & 68.56 \\
\hline 35 & 27-02 Sept & 36.7 & 40.6 & 0.0 & 91.0 & 0.0 & 33.66 & 37.45 & 111.26 \\
\hline 36 & 03-09 Sep & 29.3 & 108.4 & 29.8 & 33.9 & 88.1 & 57.9 & 37.57 & 64.89 \\
\hline 37 & 10-16 Sep & 50.0 & 28.4 & 84.5 & 7.4 & 38.4 & 41.74 & 28.57 & 68.45 \\
\hline 38 & 17-23 Sep & 35.0 & 61.9 & 150.6 & 3.4 & 57.4 & 61.66 & 54.85 & 88.96 \\
\hline 39 & 24-30 Sep & 10.0 & 21.8 & 0.0 & 0.0 & 0.0 & 6.36 & 9.66 & 151.89 \\
\hline 40 & 01-07 Oct. & 16.4 & 49.0 & 40.8 & 1.6 & 1.8 & 21.92 & 22.01 & 100.41 \\
\hline 41 & 08-14 Oct. & 2.8 & 0.0 & 66.2 & 13.8 & 0.0 & 16.56 & 28.33 & 171.07 \\
\hline 42 & 15-21 Oct. & 0.0 & 0.0 & 0.0 & 0.0 & 0.0 & 0.0 & 0.0 & 0.0 \\
\hline
\end{tabular}




\begin{tabular}{|c|c|c|c|c|c|c|c|c|c|}
\hline $\mathbf{4 3}$ & 22-28 Oct. & 0.0 & 0.0 & 10.3 & 9.2 & 0.0 & 3.9 & 5.35 & 137.18 \\
\hline $\mathbf{4 4}$ & 29-04 Nov. & 0.0 & 3.2 & 0.0 & 0.0 & 0.0 & 0.64 & 1.43 & 223.44 \\
\hline $\mathbf{4 5}$ & 05-11 Nov. & 0.0 & 1.0 & 0.0 & 2.2 & 0.0 & 0.64 & 0.97 & 151.56 \\
\hline $\mathbf{4 6}$ & 12-18 Nov. & 0.0 & 0.0 & 0.0 & 10.8 & 0.0 & 2.16 & 4.83 & 223.61 \\
\hline $\mathbf{4 7}$ & 19-25 Nov. & 0.0 & 0.0 & 14.0 & 0.0 & 0.0 & 2.80 & 6.26 & 223.57 \\
\hline $\mathbf{4 8}$ & 26-02 Dec. & 0.0 & 0.0 & 0.0 & 0.0 & 0.0 & 0.0 & 0.0 & 0.0 \\
\hline $\mathbf{4 9}$ & 03-09 Dec. & 0.0 & 0.0 & 26.6 & 0.0 & 0.0 & 5.32 & 11.90 & 223.68 \\
\hline $\mathbf{5 0}$ & 10-16 Dec. & 0.0 & 0.0 & 0.0 & 0.0 & 0.0 & 0.0 & 0.0 & 0.0 \\
\hline $\mathbf{5 1}$ & 17-23 Dec. & 0.0 & 0.0 & 0.0 & 0.0 & 0.0 & 0.0 & 0.0 & 0.0 \\
\hline $\mathbf{5 2}$ & 24-31 Dec. & 0.0 & 0.0 & 0.0 & 0.0 & 0.0 & 0.0 & 0.0 & 0.0 \\
\hline & Total & $\mathbf{6 7 7 . 5}$ & $\mathbf{6 8 8 . 2}$ & $\mathbf{1 2 0 7 . 3}$ & $\mathbf{5 6 9 . 7}$ & $\mathbf{5 7 4 . 8}$ & $\mathbf{7 4 3 . 5}$ & - & - \\
\hline
\end{tabular}

Table.2 Mean Monthly Average Rainfall of Parbhani (In mm)

\begin{tabular}{|c|c|c|c|c|c|c|c|c|}
\hline \multirow[t]{2}{*}{ Month } & \multicolumn{5}{|c|}{ Year } & \multirow[t]{2}{*}{ Mean } & \multirow[t]{2}{*}{ S.D } & \multirow[t]{2}{*}{ CV (\%) } \\
\hline & 2011 & 2012 & 2013 & 2014 & 2015 & & & \\
\hline January & 00.0 & 00.0 & 01.0 & 00.0 & 09.2 & 2.04 & 4.03 & 197.55 \\
\hline February & 00.0 & 00.0 & 22.7 & 24.4 & 00.0 & 9.42 & 12.91 & 137.05 \\
\hline March & 01.7 & 00.0 & 08.6 & 77.1 & 40.9 & 25.66 & 33.17 & 129.27 \\
\hline April & 23.4 & 00.6 & 00.0 & 07.2 & 91.8 & 24.6 & 38.73 & 157.44 \\
\hline May & 16.4 & 05.4 & 00.0 & 25.3 & 24.8 & 14.38 & 11.39 & 79.21 \\
\hline June & 36.3 & 82.5 & 202.5 & 51.5 & 125.6 & 99.68 & 66.85 & 67.06 \\
\hline July & 259.9 & 231.3 & 421.2 & 102.0 & 13.6 & 205.6 & 156.28 & 76.01 \\
\hline August & 189.3 & 71.1 & 128.5 & 176.5 & 83.2 & 129.72 & 53.24 & 41.04 \\
\hline September & 131.3 & 244.1 & 264.9 & 68.1 & 183.9 & 178.46 & 80.95 & 45.36 \\
\hline October & 19.2 & 49.0 & 117.3 & 24.6 & 01.8 & 42.38 & 45.16 & 106.56 \\
\hline November & 00.0 & 04.2 & 14.0 & 13.0 & 00.0 & 6.24 & 6.85 & 109.78 \\
\hline December & 00.0 & 00.0 & 26.6 & 00.0 & 00.0 & 5.32 & 11.90 & 223.68 \\
\hline Total & 677.5 & 688.2 & 1207.3 & 569.7 & 574.8 & 743.5 & 265.14 & 35.66 \\
\hline
\end{tabular}

Table.3 Mean Monthly Average Maximum temperature

\begin{tabular}{|c|c|c|c|c|c|c|c|c|}
\hline \multirow[b]{2}{*}{ Month } & \multicolumn{5}{|c|}{ Year } & \multirow[b]{2}{*}{ Mean } & \multirow[b]{2}{*}{ S.D } & \multirow{2}{*}{$\begin{array}{l}\text { CV } \\
(\%)\end{array}$} \\
\hline & 2011 & 2012 & 2013 & 2014 & 2015 & & & \\
\hline January & 29.85 & 30.04 & 30.86 & 29.86 & 28.95 & 29.91 & 0.68 & 2.27 \\
\hline February & 32.35 & 33.33 & 32.84 & 31.22 & 33.30 & 32.61 & 0.87 & 2.67 \\
\hline March & 37.00 & 37.34 & 37.25 & 33.66 & 34.65 & 35.98 & 1.71 & 4.75 \\
\hline April & 38.93 & 40.32 & 39.94 & 39.06 & 37.87 & 39.22 & 0.96 & 2.45 \\
\hline May & 41.09 & 41.78 & 42.75 & 39.87 & 41.75 & 41.45 & 1.06 & 2.56 \\
\hline June & 36.03 & 37.30 & 33.12 & 38.60 & 35.63 & 36.14 & 2.05 & 5.67 \\
\hline July & 31.79 & 31.57 & 29.44 & 33.25 & 35.28 & 32.27 & 2.17 & 6.72 \\
\hline August & 30.60 & 31.24 & 29.20 & 32.28 & 31.70 & 31.00 & 1.18 & 3.81 \\
\hline September & 31.18 & 31.07 & 31.98 & 31.00 & 32.48 & 31.54 & 0.66 & 2.09 \\
\hline October & 33.67 & 32.39 & 31.57 & 33.84 & 35.03 & 33.30 & 1.35 & 4.05 \\
\hline November & 32.13 & 30.79 & 30.35 & 32.15 & - & 31.36 & 0.92 & 2.93 \\
\hline December & 30.52 & 30.83 & 29.05 & 29.40 & - & 29.95 & 0.86 & 2.87 \\
\hline
\end{tabular}


Table.4 Mean Monthly minimum average Temperature

\begin{tabular}{|c|c|c|c|c|c|c|c|c|}
\hline & \multicolumn{9}{|c|}{ Year } & & CV (\%) \\
\hline Month & $\mathbf{2 0 1 1}$ & $\mathbf{2 0 1 2}$ & $\mathbf{2 0 1 3}$ & $\mathbf{2 0 1 4}$ & $\mathbf{2 0 1 5}$ & Mean & S.D & \\
\hline January & $\mathbf{9 . 5 1}$ & 12.25 & 13.73 & 12.95 & 11.41 & 11.97 & 1.62 & 13.53 \\
\hline February & 13.59 & 14.22 & 15.80 & 13.18 & 13.77 & 14.11 & 1.01 & 7.16 \\
\hline March & 16.88 & 15.56 & 18.15 & 17.81 & 18.09 & 17.30 & 1.10 & 6.36 \\
\hline April & 21.93 & 23.48 & 22.97 & 20.80 & 20.27 & 21.89 & 1.37 & 6.26 \\
\hline May & 25.51 & 26.00 & 26.85 & 23.17 & 24.93 & 25.29 & 1.38 & 5.46 \\
\hline June & 24.71 & 25.51 & 23.36 & 25.52 & 24.37 & 24.69 & 0.90 & 3.65 \\
\hline July & 23.19 & 23.25 & 22.43 & 23.09 & 24.57 & 23.31 & 0.78 & 3.35 \\
\hline August & 22.39 & 22.38 & 22.25 & 21.80 & 23.00 & 22.36 & 0.43 & 1.92 \\
\hline September & 21.80 & 22.13 & 22.40 & 20.52 & 22.17 & 21.80 & 0.75 & 3.44 \\
\hline October & 18.52 & 18.30 & 20.69 & 18.98 & 19.53 & 19.20 & 0.96 & 5.00 \\
\hline November & 13.68 & 19.02 & 13.95 & 15.56 & - & 15.55 & 2.46 & $\mathbf{1 5 . 8 2}$ \\
\hline December & 10.67 & 12.70 & 10.76 & 9.98 & - & 11.03 & 1.17 & 10.61 \\
\hline
\end{tabular}

Table.5 Mean Monthly Average Minimum Relative Humidity

\begin{tabular}{|c|c|c|c|c|c|c|c|c|}
\hline \multirow[b]{2}{*}{ Month } & \multicolumn{5}{|c|}{ year } & \multirow[b]{2}{*}{ Mean } & \multirow[b]{2}{*}{ S.D } & \multirow[t]{2}{*}{ CV (\%) } \\
\hline & 2011 & 2012 & 2013 & 2014 & 2015 & & & \\
\hline January & 30.32 & 28.32 & 31.87 & 35.71 & 31.77 & 31.60 & 2.71 & 8.58 \\
\hline February & 29.25 & 23.79 & 31.32 & 29.82 & 21.61 & 27.16 & 4.21 & 15.50 \\
\hline March & 18.81 & 12.39 & 18.58 & 35.13 & 28.68 & 22.72 & 9.06 & 39.88 \\
\hline April & 20.27 & 15.13 & 17.47 & 19.63 & 24.73 & 19.45 & 3.58 & 18.41 \\
\hline May & 19.77 & 15.97 & 15.77 & 23.48 & 19.10 & 18.82 & 3.17 & 16.84 \\
\hline June & 42.13 & 35.27 & 58.53 & 31.57 & 45.87 & 42.67 & 10.49 & 24.58 \\
\hline July & 62.00 & 63.35 & 70.94 & 55.32 & 42.20 & 58.76 & 10.79 & 18.36 \\
\hline August & 66.71 & 62.45 & 67.74 & 60.55 & 58.03 & 63.10 & 4.10 & 6.50 \\
\hline September & 61.17 & 65.00 & 58.33 & 60.63 & 55.37 & 60.10 & 3.57 & 5.94 \\
\hline October & 36.77 & 39.35 & 57.58 & 38.39 & 34.35 & 41.29 & 9.30 & 22.52 \\
\hline November & 28.87 & 37.40 & 38.87 & 34.37 & - & 34.88 & 4.42 & 12.67 \\
\hline December & 28.32 & 31.52 & 33.77 & 29.10 & - & 30.68 & 2.47 & 8.05 \\
\hline
\end{tabular}

Table.6 Mean Monthly Average Evaporation (mm)

\begin{tabular}{|c|c|c|c|c|c|c|c|c|}
\hline & \multicolumn{9}{|c|}{ Year } & & & & CV \\
\hline Month & $\mathbf{2 0 1 1}$ & $\mathbf{2 0 1 2}$ & $\mathbf{2 0 1 3}$ & $\mathbf{2 0 1 4}$ & $\mathbf{2 0 1 5}$ & Mean & S.D & $(\%)$ \\
\hline January & 4.27 & 4.81 & 5.09 & 4.47 & 4.70 & 4.69 & 0.31 & 6.61 \\
\hline February & 5.65 & 6.16 & 6.17 & 5.55 & 6.91 & 6.09 & 0.54 & 8.87 \\
\hline March & 7.69 & 9.03 & 9.15 & 6.71 & 7.73 & 8.06 & 1.02 & 12.66 \\
\hline April & 9.50 & 11.61 & 11.23 & 9.26 & 9.03 & 10.13 & 1.20 & 11.85 \\
\hline May & 11.79 & 14.52 & $\mathbf{1 5 . 1 5}$ & 10.86 & 12.60 & 12.98 & 1.81 & 13.95 \\
\hline June & 8.30 & 9.68 & 6.04 & 10.29 & 7.23 & 8.31 & 1.74 & 20.94 \\
\hline July & 4.63 & 4.08 & 3.85 & 6.18 & 9.20 & 5.59 & 2.21 & $\mathbf{3 9 . 5 3}$ \\
\hline August & 3.97 & 4.44 & 3.71 & 5.15 & 5.48 & 4.55 & 0.75 & 16.48 \\
\hline September & 4.63 & 4.05 & 4.73 & 4.79 & 5.58 & 4.76 & 0.55 & 11.55 \\
\hline October & 5.77 & 5.09 & 4.63 & 6.69 & 7.04 & 5.84 & 1.02 & 17.47 \\
\hline November & 5.85 & 4.55 & 5.00 & 5.21 & - & 5.15 & 0.54 & 10.50 \\
\hline December & 4.88 & 4.91 & 4.41 & 4.81 & - & 4.75 & 0.23 & 4.84 \\
\hline
\end{tabular}


Table.7 Mean Monthly Average Bright Sunshine Hours (hr)

\begin{tabular}{|c|c|c|c|c|c|c|c|c|c|}
\hline & \multicolumn{9}{|c|}{ Year } \\
\hline Month & $\mathbf{2 0 1 1}$ & $\mathbf{2 0 1 2}$ & $\mathbf{2 0 1 3}$ & $\mathbf{2 0 1 4}$ & $\mathbf{2 0 1 5}$ & Mean & S.D & $\begin{array}{c}\text { CV } \\
(\%)\end{array}$ \\
\hline January & 9.65 & 9.54 & 8.36 & 7.94 & 7.95 & 8.69 & 0.85 & 9.78 \\
\hline February & 10.05 & 9.86 & 8.69 & 9.44 & 9.26 & 9.46 & 0.53 & 5.60 \\
\hline March & 10.47 & 10.47 & 8.95 & 8.54 & 8.45 & 9.38 & 1.02 & 10.87 \\
\hline April & $\mathbf{1 0 . 5 8}$ & 9.80 & 9.95 & 9.19 & 9.17 & 9.74 & 0.59 & 6.06 \\
\hline May & 9.65 & 9.81 & 9.64 & 9.01 & 8.97 & 9.42 & 0.39 & 4.14 \\
\hline June & 6.97 & 7.44 & 4.28 & 7.27 & 6.07 & 6.41 & 1.30 & 20.28 \\
\hline July & 4.59 & 3.20 & 2.70 & 4.31 & 6.08 & 4.18 & 1.32 & 31.58 \\
\hline August & 3.93 & 4.23 & 3.01 & 5.97 & 5.00 & 4.43 & 1.12 & 25.28 \\
\hline September & 6.55 & 6.05 & 6.28 & 5.59 & 6.92 & 6.28 & 0.50 & 7.96 \\
\hline October & 9.33 & 7.50 & 6.87 & 7.76 & 8.39 & 7.97 & 0.94 & 11.79 \\
\hline November & 9.70 & 8.13 & 8.38 & 7.83 & - & 8.51 & 0.82 & 9.64 \\
\hline December & 9.74 & 9.32 & 8.84 & 8.31 & - & 9.05 & 0.62 & 6.85 \\
\hline
\end{tabular}

Table.8 Mean Monthly Average Wind Speed (Km/Hr)

\begin{tabular}{|c|c|c|c|c|c|c|c|c|}
\hline \multirow[b]{2}{*}{ Month } & \multicolumn{5}{|c|}{ year } & \multirow{2}{*}{ Mean } & \multirow{2}{*}{ S.D } & \multirow[t]{2}{*}{ CV $(\%)$} \\
\hline & 2011 & 2012 & 2013 & 2014 & 2015 & & & \\
\hline January & 2.93 & 3.60 & 3.55 & 3.78 & 3.95 & 3.56 & 0.39 & 10.96 \\
\hline February & 3.56 & 3.76 & 3.97 & 4.35 & 4.41 & 4.01 & 0.37 & 9.23 \\
\hline March & 4.06 & 3.76 & 4.02 & 4.68 & 5.08 & 4.32 & 0.54 & 12.5 \\
\hline April & 5.58 & 4.45 & 5.28 & 4.13 & 4.65 & 4.82 & 0.60 & 12.45 \\
\hline May & 7.03 & 7.48 & 7.59 & 5.44 & 6.57 & 6.82 & 0.87 & 12.76 \\
\hline June & 9.05 & 7.75 & 6.39 & 8.11 & 6.75 & 7.61 & 1.07 & 14.06 \\
\hline July & 6.26 & 5.82 & 5.58 & 8.08 & 8.91 & 6.93 & 1.48 & 21.36 \\
\hline August & 4.59 & 5.07 & 5.10 & 5.33 & 6.20 & 5.26 & 0.59 & 11.22 \\
\hline September & 4.09 & 3.73 & 3.67 & 4.25 & 4.19 & 4.00 & 0.27 & 6.75 \\
\hline October & 3.53 & 3.30 & 3.46 & 3.96 & 3.21 & 3.49 & 0.29 & 8.31 \\
\hline November & 3.81 & 2.91 & 3.26 & 3.34 & - & 3.33 & 0.37 & 11.11 \\
\hline December & 2.56 & 3.27 & 2.83 & 3.85 & - & 3.13 & 0.56 & 17.89 \\
\hline
\end{tabular}

Fig.1 Graphical presentation of weekly average rainfall data

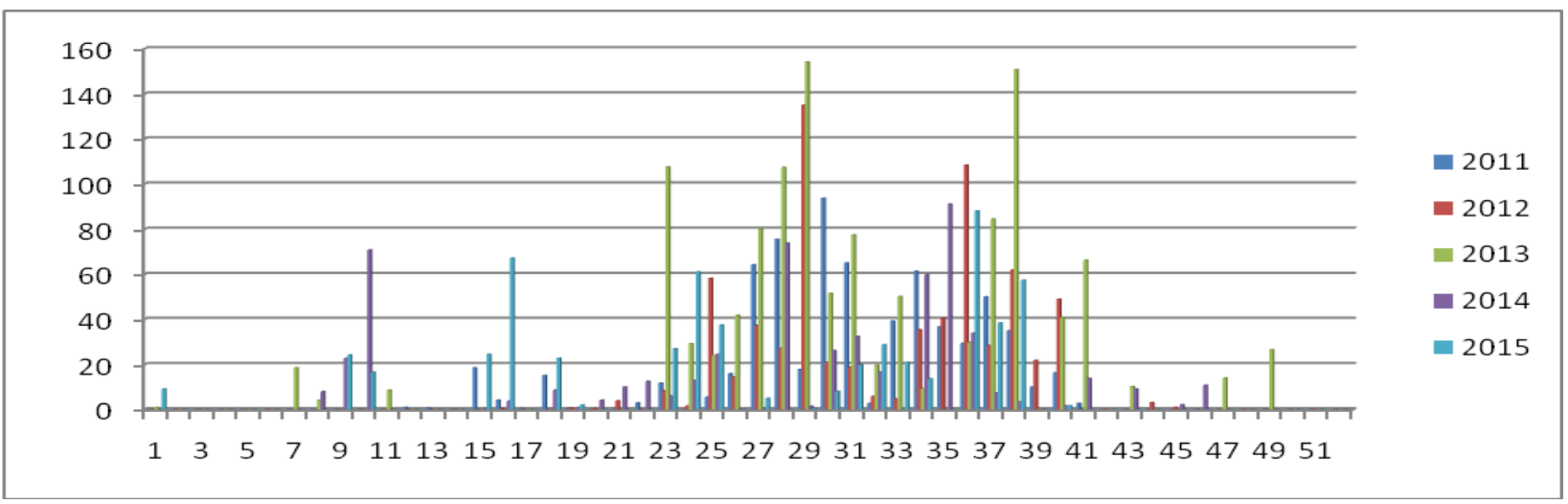




\section{Monthly Maximum Average Relative Humidity (RH)}

The table 5 indicates that March month of 2012 recorded lowest monthly minimum average $\mathrm{RH}$ in last five years with $12.39 \%$. $39.88 \%$ of highest $\mathrm{CV}$ was also recorded in March month.

\section{Mean Monthly Average Evaporation}

Table 6 shows that May month of 2013 recorded highest monthly average evaporation with $15.15 \mathrm{~mm}$ and August month of 2013 recorded lowest monthly average evaporation of $3.71 \mathrm{~mm}$ in last 5 years. Highest coefficient of Variance was recorded in July month with $39.53 \%$.

\section{Monthly Average Bright Sunshine Hours (BSS)}

From the table 7, it can be observed that April month of 2011 recorded highest monthly average BSS and July month of 2013 recorded lowest monthly average BSS. They recorded 10.58 and 2.70 hours respectively. July month recorded highest CV of $31.58 \%$ in last 5 years.

\section{Monthly Average Wind Speed}

The table 8 indicates that June month of 2011 recoded highest monthly average wind speed of $9.05 \mathrm{~km} / \mathrm{hr}$ and lowest monthly average wind speed of $2.56 \mathrm{~km} / \mathrm{hr}$ was observed in December month of the same year.

In general, July month recorded highest coefficient of Variance of $21.36 \%$ in last five years. Above results are in accordance with the results of Mohd Asim and Satyendra Nath (2015).

The daily meteorological data for Parbhani station was collected from Department of
Meteorology, Vasanthrao Naik Krishi Vidyapeeth for last five years (2011 -2015). The daily rainfall data was arranged and grouped into weekly, monthly, seasonal and annual basis while all other remaining parameters like maximum temperature, minimum temperature, minimum relative humidity, maximum relative humidity, bright sun shine hours, evaporation and wind speed were converted into monthly basis from January to December all over the period from 2011 to 2015. Average, standard deviation and coefficient of variance was calculated for each parameter.

Based on the results of the study following conclusions are drawn

The maximum average weekly rainfall of Parbhani was $154 \mathrm{~mm}$ recorded during 2013 in $29^{\text {th }}$ meteorological week.

The maximum average monthly rainfall of 421.2mm was recorded in July during 2013.

Monsoon season of 2013 recorded maximum average rainfall with $1017.1 \mathrm{~mm}$ when compared with all other seasons.

Annual average maximum rainfall of $1207.3 \mathrm{~mm}$ was observed in 2013 and minimum average annual rainfall of $569.7 \mathrm{~mm}$ was recorded during 2014 with a mean average annual rainfall of $743.5 \mathrm{~mm}$.

May month of 2013 recorded maximum average monthly temperature of $42.75^{\circ} \mathrm{C}$ and minimum average monthly temperature of $9.51^{\circ} \mathrm{C}$ was recorded in January, 2011 when compared with all other months of entire study period from 2011 to 2015 .

Maximum average monthly relative humidity of $91.17 \%$ was recorded in September month of 2012 while minimum RH was recorded in March month with $12.39 \%$ of the same year. 
May month of 2013 recorded maximum evaporation rate with $15.15 \mathrm{~mm}$ while August month of the same year recorded minimum evaporation rate of $3.71 \mathrm{~mm}$ in the entire study period.

Maximum average monthly bright sun shine hours were recorded in April, 2011 with $10.58 \mathrm{hrs}$ and minimum was recorded in the month of July, 2013 with $2.71 \mathrm{hrs}$.

June month of 2011 recorded maximum average monthly wind speed of $9.05 \mathrm{kmph}$ while lowest was observed in December month of the same year with $2.56 \mathrm{kmph}$.

\section{References}

Biswas B.G and S. D. Maskel (1981). Rainfall analysis for use in Dryland Agriculture. Indian J. Soil Conservation. Vol 9(2): 819.

Cannarozzo, M., L. V. Noto, and F. Viola. (2006). Spatial distribution of rainfall trends in Sicily (1921-2000). Physics and Chemistry of the Earth, Parts A/B/C 31(18): 1201-1211.

Ceballos-Barbancho, A., E. Morán-Tejeda, M. A. Luengo-Ugidos, and J. M. Llorente-Pinto. (2008). Water resources and environmental change in a Mediterranean environment: the southwest sector of the Duero river basin (Spain). Journal of Hydrology 351(2):126-138

Jestinos Mzezewa1, Titus Misi and Leon D van Rensburg (2010). Characterisation of rainfall at a semi-arid ecotope in the Limpopo Province (South Africa) and its implications for sustainable crop production. Water SA Vol. 36 No. 1, pp: $19-26$.

Mohd Asim and Satyendra Nath (2015). Study on Rainfall Probability Analysis at Allahabad District of Uttar Pradesh. Journal of Biology, Agriculture and Healthcare. Vol.5, No.11, pp: 214 222.

\section{How to cite this article:}

Pooran Pragnya, Tapre Praveen and Bhuibhar B. W. 2018. Analysis of Rainfall and Other Weather Parameters under Climatic Variability of Parbhani (2011-2015). Int.J.Curr.Microbiol.App.Sci. 7(06): 2487-2495. doi: https://doi.org/10.20546/ijcmas.2018.706.295 